\title{
Clinical predictors at admission and combined treatment for COVID-19 outcomes
}

\author{
Maiara Floriani ${ }^{1}$, Marina Bessel ${ }^{1}$, Isabelle Zorzo ${ }^{1}$, Andressa Glaeser ${ }^{1}$, Rafael Grando ${ }^{1}$, \\ Raphael Rodeghiero ${ }^{1}$, Mohamed Parrini ${ }^{1}$, Gisele Nader Bastos ${ }^{1}$, and Luiz Nasi ${ }^{1}$ \\ ${ }^{1}$ Hospital Moinhos de Vento
}

February 8, 2021

\begin{abstract}
Aims of the study: To investigate the effect of clinical predictors on admission and the set of therapeutic interventions on length of stay, ICU admission, need for MV and mortality. Methods used to conduct the study: Retrospective cohort of inpatients with RT-PCR positive for COVID-19 from March to July 2020. Multivariate models were used to assess risk for ICU admission, need for MV and hospital mortality. Logistic regression analysis was conducted to examine factors associated with the results. Results of the study: 459 patients were enrolled (median age 60.0 years old). For patients treated with AZMCorticosteroid (46.8\%) the risk for ICU admission was 0.17 (OR; 95\%CI 0.05-0.57), for MV 0.16 (OR; 95\%CI 0.04-0.74) and for mortality 0.16 (OR; 95\%CI 0.03-0.91). AZM-Corticosteroid also decreased the length of stay in 1.5 day. AZM-Corticosteroid and anticoagulation when indicated (17.2\%), also reduced the ICU stay in 1.5 and MV in 4 days. When included HCQ, the benefits were lost and the times increased. Age $>65$ years, presence of up one comorbidity, pulmonary involvement more than or equal to $50 \%$, saturation $<93 \%$, lymphocytes $<900 \mathrm{~mm} 3$, D-dimers $>1,250 \mathrm{ng} / \mathrm{mL}$ and $\mathrm{CRP}>8.0 \mathrm{mg} / \mathrm{dL}$ at admission were clinical predictors for death. HFNC was able to prevent intubation by $38.1 \%$. Conclusion drawn from the study and clinical implications: AZM-Corticosteroids and anticoagulation represented a favorable combination for inpatients with COVID-19, reducing length of hospitalization, risk of MV and mortality. HCQ did not yield benefits to combination therapy and we do not support its use for inpatients. HFNC was able to prevent intubation in one third of patients. Already on admission some clinical predictors may help to estimate a higher risk of poor evolution.
\end{abstract}

\section{Clinical predictors at admission and combined treatment for COVID-19 outcomes}

Running title: Clinical predictors and treatment for COVID-19

\section{Authors:}

Maiara Anschau Floriani* MSc ${ }^{1}$ (Floriani MA - maiara.floriani@hmv.org.br)

Marina Bessel ScD ${ }^{2}$ (Bessel M - marina.bessel@hmv.org.br)

Isabelle Weschenfelder Zorzo BSHCA ${ }^{1}$ (Zorzo IW - isabelle.zorzo@hmv.org.br)

Andressa Barreto Glaeser BSc${ }^{1}$ (Glaeser AB - andressa.glaeser@hmv.org.br)

Rafael Domingos Grando $\mathrm{MD}^{3}$ (Grando RD - rdgrando@hotmail.com)

Raphael Goveia Rodeghiero $\mathrm{MD}^{3}$ (Rodeghiero RG - raphael.rodeghiero@hmv.org.br)

Mohamed Mutlaq Parrini BEcon MPhil ${ }^{4}$ (Parrini MM - mohamed.parrini@hmv.org.br)

Gisele Alsina Nader Bastos MD ScD ${ }^{5}$ (Bastos GAN - gisele.nader@hmv.org.br)

Luiz Antonio Nasi MD ScD ${ }^{6}$ (Nasi LA - luiz.nasi@hmv.org.br) 


\section{Affiliations:}

${ }^{1}$ Value Management Office, Medical Manager at Hospital Moinhos de Vento, Porto Alegre, Rio Grande do Sul, Brazil

${ }^{2}$ PROADI-SUS Project Office, Hospital Moinhos de Vento, Porto Alegre, Rio Grande do Sul, Brazil

${ }^{3}$ Department of Radiology at Hospital Moinhos de Vento, Porto Alegre, Rio Grande do Sul, Brazil

${ }^{4}$ Chief Executive Officer at Hospital Moinhos de Vento, Porto Alegre, Rio Grande do Sul, Brazil

${ }^{5}$ Medical Manager, Hospital Moinhos de Vento, Porto Alegre, Rio Grande do Sul, Brazil

${ }^{6}$ Chief Medical Officer, Hospital Moinhos de Vento, Porto Alegre, Rio Grande do Sul, Brazil.

Correspondence to: Maiara Anschau Floriani

Hospital Moinhos de Vento - Moinhos Research Institute

910 Ramiro Barcelos Street, 5 floor.

Floresta - Porto Alegre - RS. 90560-030, Brazil

Conflict of Interest: The authors declare that there is no conflict of interest.

\section{ABSTRACT}

Aims of the study: To investigate the effect of clinical predictors on admission and the set of therapeutic interventions on length of stay, ICU admission, need for MV and mortality.

Methods used to conduct the study: Retrospective cohort of inpatients with RT-PCR positive for COVID-19 from March to July 2020. Multivariate models were used to assess risk for ICU admission, need for MV and hospital mortality. Logistic regression analysis was conducted to examine factors associated with the results.

Results of the study: 459 patients were enrolled (median age 60.0 years old). For patients treated with AZM-Corticosteroid (46.8\%) the risk for ICU admission was 0.17 (OR; 95\%CI 0.05-0.57), for MV 0.16 (OR; 95\%CI 0.04-0.74) and for mortality 0.16 (OR; 95\%CI 0.03-0.91). AZM-Corticosteroid also decreased the length of stay in 1.5 day. AZM-Corticosteroid and anticoagulation when indicated (17.2\%), also reduced the ICU stay in 1.5 and MV in 4 days. When included HCQ, the benefits were lost and the times increased. Age $>65$ years, presence of up one comorbidity, pulmonary involvement more than or equal to $50 \%$, saturation $<93 \%$, lymphocytes $<900 \mathrm{~mm}^{3}$, D-dimers $>1,250 \mathrm{ng} / \mathrm{mL}$ and CRP $>8.0 \mathrm{mg} / \mathrm{dL}$ at admission were clinical predictors for death. HFNC was able to prevent intubation by $38.1 \%$.

Conclusion drawn from the study and clinical implications:AZM-Corticosteroids and anticoagulation represented a favorable combination for inpatients with COVID-19, reducing length of hospitalization, risk of MV and mortality. HCQ did not yield benefits to combination therapy and we do not support its use for inpatients. HFNC was able to prevent intubation in one third of patients. Already on admission some clinical predictors may help to estimate a higher risk of poor evolution.

What's known? Studies show the ineffectiveness of HCQ in the therapeutic context of COVID-19.

What's new? We were able to describe clinical predictors of the patient's arrival at the hospital associated with the worst outcomes for the evaluated outcomes. Moreover, considering that it is a real-life study, we demonstrated the combination of favorable treatment related to decreased length of stay and risk for ICU admission, need for MV and mortality.

Keywords: COVID-19; treatment effective; cohort Brazil; hydroxychloroquine; azithromycin.

Abbreviations: ICU, intensive care unit; MV, mechanical ventilation; AZM, azithromycin; HCQ, hydroxychloroquine; HFNC, high flow nasal cannula; CRP, C-reactive protein; 


\section{INTRODUCTION}

Doctors and hospitals have learned a lot about how best to treat people infected with the novel coronavirus disease 2019 (COVID-19) in the last months since the pandemic began. A significant percentage of patients develop health conditions that require hospital care ${ }^{1}$. The practice has varied widely across the world and several therapeutic interventions have been proposed and methodological studies have been published, although far from overwhelming evidence they closely follow and analyze updates on this outbreak ${ }^{2}$, but there is no consensus on the best decisions.

Therapeutic strategies using hydroxychloroquine (HCQ), antibiotics, corticosteroid, anticoagulants and others, in combination or not, were introduced to the clinical practice. However, there is not yet consensus about the best pharmacological combination to prove effectiveness and safety, incorporated in the usual care in the COVID-19 treatment. Despite that, recently a Brazilian guideline ${ }^{3}$ recommended a number of therapeutic strategies in the management of COVID-19 patients based on available scientific evidence, discouraging the use of HCQ.

Our objective was to retrospectively evaluate medical practice in the real world, considering the impact of clinical predictors evaluated on the arrival and the use of different therapeutic combinations on the length of stay, the need for admission to the intensive care unit (ICU) or mechanical ventilation (MV) and mortality during the outbreak of COVID-19 in our center.

\section{METHODS}

Observational retrospective analysis of patients (aged [?]18 years) with RT-PCR positive for COVID-19 obtained from nasal and pharyngeal swabs, admitted from March 15 to July 31, 2020 at Hospital Moinhos de Vento, a teaching private hospital, with 500 beds and reference for the treatment of patients with COVID19 in the South of Brazil ${ }^{4}$.

Outcomes of different treatment combinations used in standard care were evaluated: HCQ, azithromycin (AZM), corticosteroids (dexamethasone, hydrocortisone or methylprednisolone), tocilizumab, oseltamivir, convalescent plasma, therapeutic anticoagulation and different ways of improving oxygen without positive pressure (nasal catheter and Hudson mask) and with positive pressure (high flow nasal cannula (HFNC), Bilevel Airway Positive Pressure (BiPAP) and MV). Therapeutic anticoagulation was considered with intentionto-treat for patients with deep venous thrombosis (DVT), pulmonary embolism (PE) or D-dimers elevated. Obese people (body mass index [?]30), lymphopenia (lymphocytes $<900 \mathrm{~mm} 3$ ), D-dimers $>1,250 \mathrm{ng} / \mathrm{mL}$ and C-reactive protein $(\mathrm{CRP})>8.0 \mathrm{mg} / \mathrm{dL}$ were considered abnormal values. The usual dose of corticosteroids used was [?]0.5-1 mg/kg per day of methylprednisolone or equivalent. The pulmonary impairment was assessed on a visual scale by two independent chest radiologists trained to interpreted COVID-19 patients.

Primary endpoint was building up a COVID-19 predictor model based on individual-level data to estimate the risk for ICU admission, need for MV and hospital mortality. Additionally, three secondary endpoints were established: 1) the impact of combined interventions on hospital length of stay, ICU and MV, 2) likelihood that different ways of noninvasive oxygen administration prevent MV and 3) which clinical predictors are relevant to poor outcome (mortality). The Institutional Ethics Committee approved this study (CAAE 30350820.5.2037.5330).

\section{Statistical Analysis}

Descriptive analysis was used to characterize the study population. Categorical variables were summarized using absolute frequencies and percentages, while continuous variables were analyzed using means and standard deviation (SD) or median and interquartile range (IQR). Interaction term with HCQ was used to evaluate the behavior of the combined therapy of medications in the presence or absence of the use of HCQ.

Logistic regression analysis was conducted to examine factors associated with the outcomes, adjusting for all predictors presented in the model. Linear regression was applied to continuous outcomes. The model results are presented in odds ratio (OR) or beta (?). For the multivariable analyses, a theoretical framework 
was structured according to literature. Statistical analysis was performed using SAS software (Statistical Analysis System, SAS Institute Inc., Cary, N.C.), version 9.4, and statistical significance was defined as p-value $<0.05$.

\section{RESULTS}

Patients admitted to Hospital Moinhos de Vento (HMV), from March 15 to July 31, 2020, were included in the study, a total of 459 individuals with a median age of 60.0 years (IQR, 45.0 to 72.0 ), being $57.1 \%$ males. Hypertension (46.8\%), pulmonary disease (emphysema, COPD or asthma) (22.1\%) and diabetes (21.6\%); fever $(63.5 \%)$, cough $(60.6 \%)$ and dyspnea (38.7) were the frequently reported comorbidities and symptoms at hospital admission. The median onset of symptoms before admission was 7.0 days (IQR, 4.0 to 9.5); 136 (29.6\%) patients needed admission to the ICU and 97 (21.1\%) required MV. The mean length of stay in hospital, MV and ICU was 13.9 (SD+-16.1), 20.1 (SD+-15.6) and 21.2 (SD+-18.4) days, respectively.

Regarding pharmacologic therapies, HCQ-AZM was administered to 105 (23.2\%) patients; HCQcorticosteroid to $49(10.7 \%)$ and AZM-corticosteroid to 212 (46.8\%). Therapeutic anticoagulation was administered to 138 (31.8\%) patients, being 36 (7.9\%) combined with HCQ and 78 (17.2\%) with AZMCorticosteroid. The combination of HCQ-AZM-Corticosteroid and therapeutic anticoagulation occurred in 21 patients $(4.6 \%)$. For patients treated with AZM-Corticosteroid the risk for ICU admission 0.17 (OR; $95 \%$ CI 0.05 to 0.57 ), for MV 0.16 (OR; 95\%CI 0.04 to 0.74 ) and mortality was 0.16 (OR; 95\% CI 0.03 to 0.91 ). For those patients treated with HCQ-AZM-Corticosteroid, the association represented loss of benefit (Figure 1).

Figure 1 Outcomes risk according to treatment combined

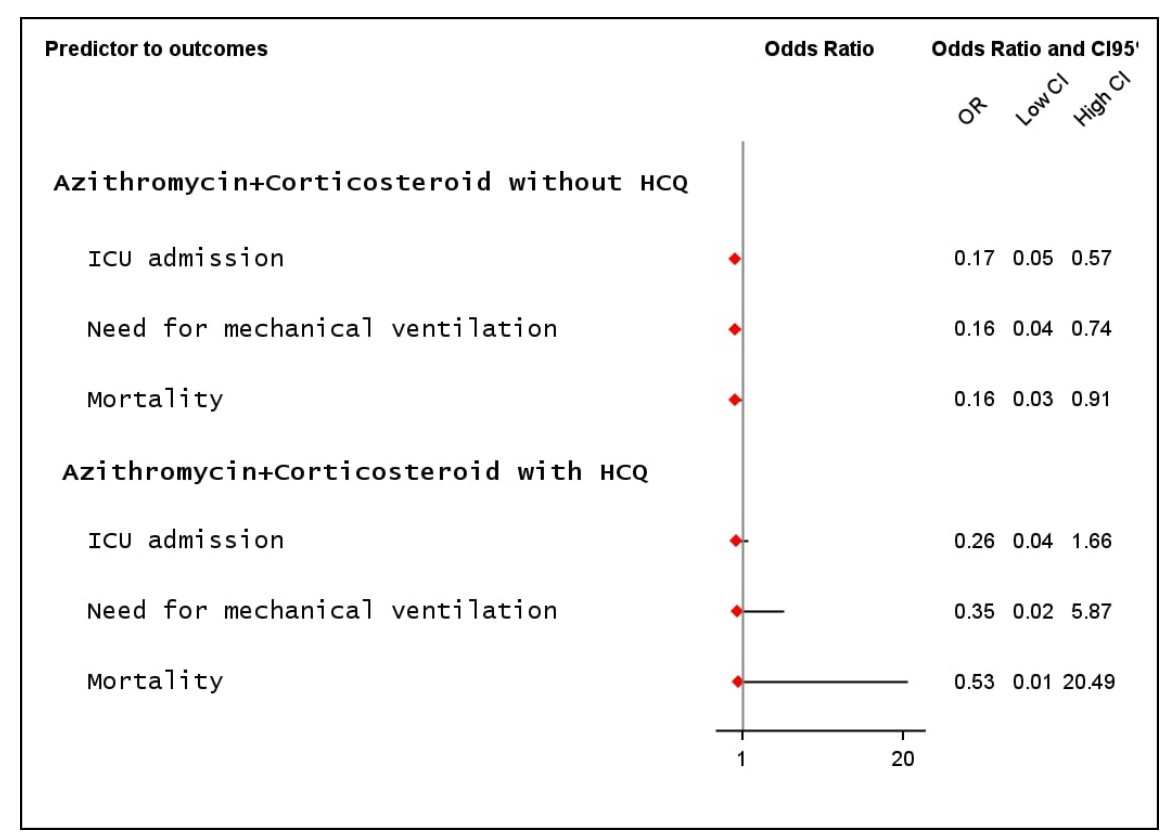

.Risk predictors to primary endpoint - ICU admission, need for MV and hospital mortality

Tocilizumab (2.6\%), convalescent plasma (16.5\%), oseltamivir $(29.6 \%)$ and others antibiotics (79.7\%), excluding AZM, were administered as adjunct therapy. Among patients admitted to the ICU, 57.6\% (68) received vasopressor and supplemental oxygen without positive pressure was used in $56.9 \%$ (254). Positive non-invasive ventilation including HFNC and BiPAP were used in 23.9\% (107) while MV in 21.1\% (97). HFNC oxygen therapy was able to prevent the patient's progression to MV in $31.8 \%$ of cases. 
Overall, $86.7 \%$ (398) were discharged alive and $4.5 \%$ (21) were still hospitalized by dataset freeze date. Of patients admitted to the ICU, $25.7 \%$ (35) died and when MV was required, the mortality increased to $34.0 \%$ (33). Among dead patients, the median age was 83.3 years (IQR, 75.5 to 89.5) with the length of stay in hospital of $25.3(\mathrm{SD} \pm 22.5)$ days, $22.8(\mathrm{SD} \pm 18.7)$ in $\mathrm{ICU}$ and $21.2(\mathrm{SD} \pm 17.3)$ in MV. The main clinical predictors related to increase the risk for mortality $(>70 \%)$, were: age $>65$ years, presence of up one comorbidity, pulmonary involvement [?]50\%, saturation $<93 \%$, lymphocyte $<900 \mathrm{~mm}^{3}$, D-dimers $>1,250 \mathrm{ng} / \mathrm{mL}$ and $\mathrm{CRP}>8.0 \mathrm{mg} / \mathrm{dL}$ at admission, oxygen requirement through BiPAP or HFNC, and ICU admission and MV required during hospitalization were also associated with a higher risk of death (Figure 2) (Table).

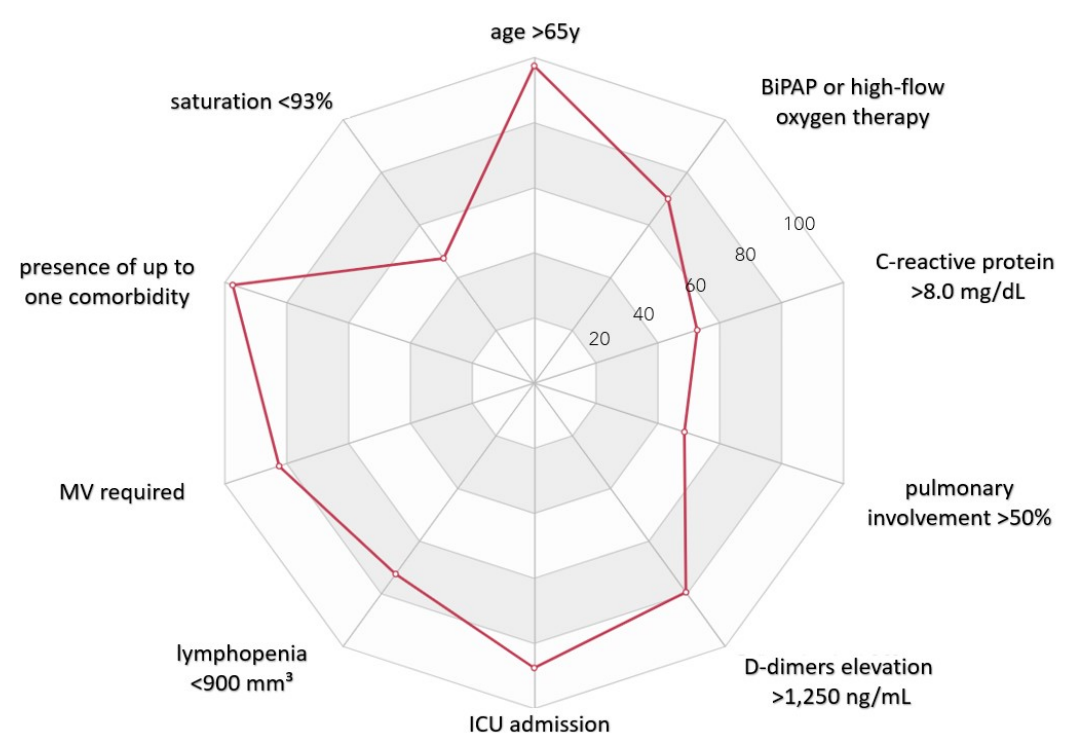

Figure 2 Clinical predictors in mortality

Table. Clinical predictors at admission and hospitalization associated with poor outcome

\begin{tabular}{lll}
\hline AT ADMISSION & n (\%) or median [IQR] & AT HOSPITALIZATION \\
Onset of symptoms and hospital admission (days) & $7.0[4.0-9.5]$ & ICU admission \\
Male & $262(57.0)$ & Mechanical ventilation (MV) \\
Age $>65$ years & $184(40.0)$ & BiPAP or High flow nasal cannula (HFNC \\
Obesity BMI [?] 30 & $148(33.1)$ & \\
Saturation $<93 \%$ & $73(16.7)$ & OUTCOMES \\
Presence of up to 1 comorbidity & $341(74.6)$ & $\begin{array}{l}\text { Hospital discharge } \\
\text { Death }\end{array}$ \\
COMPROMISE LUNG (chest CT) & & Censored* \\
$50 \%$ & $68(16.2)$ & \\
LABORATORY & & \\
D-dimers $\left(>1,250 \mathrm{ng} / \mathrm{mL}^{*}\right)$ & $71(17.5)$ & \\
C-reactive protein $(>8.0 \mathrm{mg} / \mathrm{dL})$ & $141(33.6)$ & \\
Lymphopenia $\left(<900 \mathrm{~mm}^{3}\right)$ & $180(39.4)$ & \\
\hline
\end{tabular}

BMI body mass index; CT computed Tomography; ICU intensive care unit; BiPAP Bi-level Airway Positive Pressure; *censored means patients without outcome results

For secondary endpoints, AZM-Corticosteroid decreased the length of hospital stay in 1.5 day (? -1.5 95\%CI 
-4.9 to 1.9), while HCQ use increased in 6.9 days (? $6.995 \%$ CI 3.6 to 10.3). AZM-Corticosteroid and therapeutic anticoagulation combination reduced the ICU length stay in 1.5 day (? $-1.595 \% \mathrm{CI}-9.7$ to 6.7) and MV in 4 days (? -4.0 95\% CI -13.4 to 5.3), however, this effect was not observed when HCQ was associated (Figure 3). For the AZM-Corticosteroid and therapeutic anticoagulation the mean length of ICU stay was 15.9 days, but when HCQ was included, this time was increased to 40.3 days (Figure 3). There was a trend to more time in MV in obese patients. All models were adjusting for sex, age, obesity, pulmonary involvement, D-dimers, CRP, oxygen support without positive pressure (nasal catheter and Hudson mask) and length of hospital stay. Regarding HCQ have been used or not, the comparability of the groups was verified in relation to the clinical predictors for mortality, where there was no difference was observed among those who received the drug or not between those treated or not with HCQ.
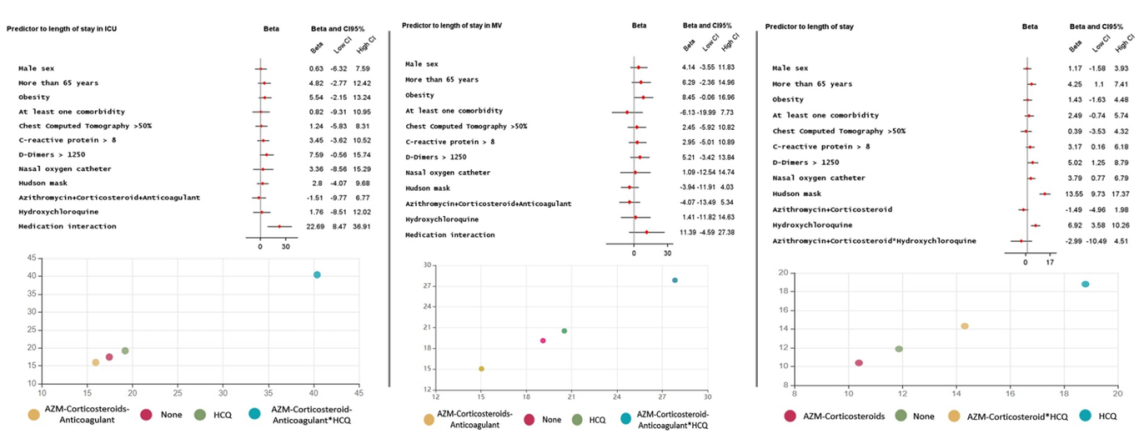

Figure 3 Risk factors associated with length of stay.

Above, the forest plot demonstrates the behavior of clinical predictors and treatments in relation to risk. Below, the chart demonstrates (colors) the behavior of combined or non-combined therapies on the mean length of stay by outcome. Medication interaction is the behavior of the combination AZM-CorticosteroidsAnticoagulant in the presence or absence of HCQ.

\section{DISCUSSION}

Our study sought to identify the clinical predictors for COVID-19 that resulted in risk for worst outcomes and the effect of combined therapeutic on length of stay, ICU admission, need for MV and mortality. Our findings suggest that 1) the combination of AZM-Corticosteroid reduced the risk of ICU admission, need for MV and mortality; 2) AZM-Corticosteroid and therapeutic anticoagulation, when indicated, reduced the mean length of stay in ICU and MV; 3) the introduction of HCQ to the AZM-Corticosteroid combination increased the mean length hospital stay; 4) the use of HFNC prevented in one third the patient's progression to MV and 5) Age $>65$ years, presence of up one comorbidity, pulmonary involvement [?] 50\%, saturation $<93 \%$, lymphopenia, D-dimers and CRP altered were admission clinical predictors associated with higher risk for mortality.

Observational and Randomized Clinical Trials (RCT) have been published, evaluating the effects of several drugs in terms of potency, efficiency or efficacy in clinical management ${ }^{3,5}$. Predictive factors and clinical characteristics that may influence COVID-19 severity have already been demonstrated in the literature and multivariable models have been used to identify high-risk individuals ${ }^{6,7}$.

Although some treatments are promising, it is thought to be early to clearly state that there is a definitive treatment. The Solidarity Trial Consortium ${ }^{8}$, funded by the World Health Organization showed that antiviral drugs including remdesivir, HCQ, lopinavir, and interferon regimens had little or no effect on hospitalized patients with COVID-19, as indicated by overall mortality, initiation of ventilation, and duration of hospital stay. Also, another promising intervention with convalescent plasma, validated in a RCT showed no significant differences in clinical status or overall mortality between patients treated or received placebo ${ }^{9}$. 
Although controversial, the use of corticosteroid seems to have clinical potential on mortality reduction and need for intubation, provided it is adequate for the treatment regimen and individual clinical characteristics $^{10-12}$. The Randomized Evaluation of COVID-19 Therapy (RECOVERY trial) showed that survival was significantly higher among patients treated with dexamethasone, especially for those requiring invasive intubation ${ }^{13}$. Our data reinforce these findings and highlight the corticosteroid therapeutic effectiveness, especially in reducing the risk of mortality, ICU admission and need for MV when combined with AZM. Also, we observed benefits related to reduced length of stay in ICU and MV in patients who used anticoagulant, findings additional to those reported by Nadkarni $(2020)^{14}$, where therapeutic or prophylactic anticoagulation reduced intubation and mortality.

Some studies published at the beginning of the pandemic, with limited evidence, highlighted the benefits of using HCQ combined or not with AZM in reducing mortality and length of stay ${ }^{15-16}$. However, in an open-label, multicenter, randomized, controlled trial conducted by the Coalition COVID-19 Brazil I, among inpatients with mild-to-moderate COVID-19, the use of HCQ, alone or with AZM, did not improve clinical status at 15 days as compared with standard care $^{17}$. Self et al. ${ }^{18}$ reported similar ineffectiveness in HCQ treatment on the 14th day of hospitalization.

Rosenberg (2020) ${ }^{19}$ and Magagnoli (2020) ${ }^{20}$ also performed a protocol using HCQ combined or not with AZM and found no reduction in mortality risk and need for MV. Also, they reported an increase in overall mortality for patients treated with HCQ alone. In the same direction, we observed that whenever HCQ was included in the model, the protective benefit of the association of AZM-Corticosteroids loses significance and becomes a risk factor for a worse prognosis. We showed that patients treated with HCQ have a longer hospitalization compared to patients not treated, a finding previously discussed by Kalligeros et $\mathrm{al}^{21}$. Interestingly also, when we analyzed the clinical predictors influence under the use of HCQ, there was no significant difference between those treated or not with HCQ. We assume that other factors, such drug interactions may be involved in these findings. Besides that, oseltamivir, convalescent plasma, vasopressor and tocilizumab when evaluated alone or combined with HCQ, AZM and corticosteroids showed no benefit.

The use of HFNC showed a trend toward reduction in the intubation rate and no difference in mortality, findings similar to those reviewed by Lin $(2020)^{22}$. Geng (2020) presented HFNC as a favorable option to avoid intubation through adequate monitoring of the respiratory function of COVID patients ${ }^{23}$.

Clinical predictors at admission associated with higher risk for mortality include individuals older than $>65$ years, with up to one comorbidity, pulmonary involvement more than $50 \%$, saturation $<93 \%$, lymphopenia, D-dimers and CRP elevated. We understand that if these clinical variables are considered upon patient arrival, management and treatment will be more effective. Oxygen requirement through BiPAP or HFNC, ICU admission and MV required during hospitalization were also risk markers. A recent publication showed that patients with leukocytosis and CRP altered on arrival were associated with poor prognosis and may predict the severity of COVID-19 ${ }^{24}$.

The weakness of our study is related to the fact that it is observational, unicentric and retrospective however our results are in line with other RCTs that recommended the association of corticosteroids to the set of treatment and advise against HCQ use in patients with COVID-19. The reduced HCQ treatment efficiency when included to the set of drugs can be speculate through the pharmacological interaction with others drugs triggering for example an increase in its serum concentration, prolongation of the QT interval in the ECG and possibly triggering episodes of ventricular tachycardia ${ }^{25-28}$. It is known that longer QTc can cause life-threatening arrhythmias especially in critically ill patients, however monitoring of ECG and drug serum level was not uniformly standardized, given the retrospective nature of the study. Finally, the results of our study should be evaluated considering individual clinical characteristics in a real world and clinicians should carefully weigh the risks and benefits when considering any therapeutic scheme out of the randomized clinical trial setting.

\section{CONCLUSION}

In this retrospective cohort, AZM-Corticosteroids and therapeutic anticoagulation, when indicated, rep- 
resented a favorable combination for patients hospitalized with COVID-19, reducing mortality, length of hospitalization and the risk for MV. HCQ did not yield benefits to combination therapy and we do not support its use for inpatients. HFNC oxygen therapy was able to reduce the risk of MV support. Individuals older than $>65$ years, with presence of up one comorbidity, pulmonary involvement more than or equal to $50 \%$, saturation $<93 \%$, lymphopenia, D-dimers and CRP elevated on arrival, and oxygen requirement through BiPAP or HFNC, ICU admission and MV during hospitalization represented the set of clinical predictors for worse prognosis.

\section{ACNOWLEDGEMENTS OF FUNDING SUPPORT}

None

\section{REFERENCES}

1. Verity R, Okell LC, Dorigatti I, et al. Estimates of the severity of coronavirus disease 2019: a modelbased analysis. Lancet Infect Dis. 2020;20:669-77.

2. Infectious Diseases Society of America Guidelines on the Treatment and Management of Patients with COVID-19. Available at: https://www.idsociety.org/practice-guideline/covid-19-guideline-treatmentand-management/. Accessed in November, 2020.

3. Falavigna M, Colpani V, Stein C, et al. Guidelines for the pharmacological treatment of COVID-19. The task-force/consensus guideline of the Brazilian Association of Intensive Care Medicine, the Brazilian Society of Infectious Diseases and the Brazilian Society of Pulmonology and Tisiology. Diretrizes para o tratamento farmacológico da COVID-19. Consenso da Associação de Medicina Intensiva Brasileira, da Sociedade Brasileira de Infectologia e da Sociedade Brasileira de Pneumologia e Tisiologia. Rev Bras Ter Intensiva.2020;32(2):166-196.

4. Polanczyk CA, Rohsig V, Bastos GN, et al. Getting Ready for the Covid-19 Pandemic: Experience of a Brazilian Hospital. $N$ Engl J Med . 2020.

5. Wise J, Coombes R. Covid-19: The inside story of the RECOVERY trial.BMJ . 2020;370:m2670.

6. Barda N, Riesel D, Akriv A, et al. Developing a COVID-19 mortality risk prediction model when individual-level data are not available. Nat Commun . 2020;11:4439.

7. Zhao Z, Anne C, Wei H, et al. Prediction model and risk scores of ICU admission and mortality in COVID-19. PloS one . 2020;15,7: e0236618.

8. WHO Solidarity Trial Consortium, Pan H, Peto R, et al. Repurposed Antiviral Drugs for Covid-19 Interim WHO Solidarity Trial Results. N Engl J Med. 2020;NEJMoa2023184.

9. Simonovich VA, Burgos Pratx LD, Scibona P, et al. PlasmAr Study Group. A Randomized Trial of Convalescent Plasma in Covid-19 Severe Pneumonia. N Engl J Med. 2020:NEJMoa2031304.

10. Fang X, Mei Q, Yang T, et al. Low-dose corticosteroid therapy does not delay viral clearance in patients with COVID-19. J Infect . 2020;81(1):147-178.

11. Shang L, Zhao J, Hu Y, Du R, Cao B. On the use of corticosteroids for 2019-nCoV pneumonia. Lancet. 2020;395(10225):683-684.

12. World Health Organization. Corticosteroids for COVID-19 guideline. Available at:https://www.who.int/publications/i/item/WHO-2019-nCoV-Corticosteroids-2020.1. Accessed in December, 2020.

13. De Backer D, Azoulay E, Vincent JL. Corticosteroids in severe COVID-19: a critical view of the evidence. Crit Care.2020;24:627.

14. Nadkarni GN, Lala A, Bagiella E, et al. Anticoagulation, Bleeding, Mortality, and Pathology in Hospitalized Patients With COVID-19. J Am Coll Cardiol. 2020;76(16):1815-1826.

15. Tanriverdİ E, ÇÖrtük M, Yildirim BZ, et al. The use of hydroxychloroquine plus azithromycin and early hospital admission are beneficial in Covid-19 patients: Turkey experience with real-life data. Turk J Med Sci. 2020.

16. Arshad S, Kilgore P, Chaudhry ZS, et al. Treatment with hydroxychloroquine, azithromycin, and combination in patients hospitalized with COVID-19. Int J Infect Di s. 2020;97:396-403.

17. Cavalcanti AB, Zampieri FG, Rosa RG, et al. Coalition Covid-19 Brazil I Investigators. Hydroxychloro- 
quine with or without Azithromycin in Mild-to-Moderate Covid-19. N Engl J Med.2020;383(21):20412052.

18. Self WH, Semler MW, Leither LM, et al. Effect of Hydroxychloroquine on Clinical Status at 14 Days in Hospitalized Patients With COVID-19: A Randomized Clinical Trial. JAMA. 2020;e2022240.

19. Rosenberg ES, Dufort EM, Udo T, et al. Association of Treatment With Hydroxychloroquine or Azithromycin With In-Hospital Mortality in Patients With COVID-19 in New York State. JAMA. 2020;323(24):2493-2502. doi:10.1001/jama.2020.8630

20. Magagnoli J, Narendran S, Pereira F, et al. Outcomes of hydroxychloroquine usage in United States veterans hospitalized with Covid-19. Preprint. medRxiv. 2020;2020.04.16.20065920.

21. Kalligeros M, Shehadeh F, Atalla E, et al. Hydroxychloroquine use in hospitalised patients with COVID-19: An observational matched cohort study. J Glob Antimicrob Resist . 2020;22:842-844.

22. Lin SM, Liu KX, Lin ZH, Lin PH. Does high-flow nasal cannula oxygen improve outcome in acute hypoxemic respiratory failure? A systematic review and meta-analysis. Respir Med . 2017;131:58-64.

23. Geng S, Mei Q, Zhu C, et al. High flow nasal cannula is a good treatment option for COVID-19. Heart Lung . 2020;49(5):444-445.

24. Yamada T, Wakabayashi M, Yamaji T, et al. Value of leukocytosis and elevated C-reactive protein in predicting severe coronavirus 2019 (COVID-19): A systematic review and meta-analysis. Clinica Chimica Acta; International Journal of Clinical Chemistry.2020;509:235-243.

25. Huang HD, Jneid H, Aziz M, et al. Safety and Effectiveness of Hydroxychloroquine and Azithromycin Combination Therapy for Treatment of Hospitalized Patients with COVID-19: A Propensity-Matched Study. Cardiol Ther . 2020;9(2):523-534.

26. Mercuro NJ, Yen CF, Shim DJ, et al. Risk of QT Interval Prolongation Associated With Use of Hydroxychloroquine With or Without Concomitant Azithromycin Among Hospitalized Patients Testing Positive for Coronavirus Disease 2019 (COVID-19). JAMA Cardiol . 2020;1;5(9):1036-1041.

27. Chorin E, Wadhwani L, Magnani S, et al. QT interval prolongation and torsade de pointes in patients with COVID-19 treated with hydroxychloroquine/azithromycin. Heart Rhythm . 2020;17(9):1425-1433.

28. Saleh M, Gabriels J, Chang D, et al. Effect of Chloroquine, Hydroxychloroquine, and Azithromycin on the Corrected QT Interval in Patients With SARS-CoV-2 Infection. Circ Arrhythm Electrophysiol . 2020;13(6):e008662.

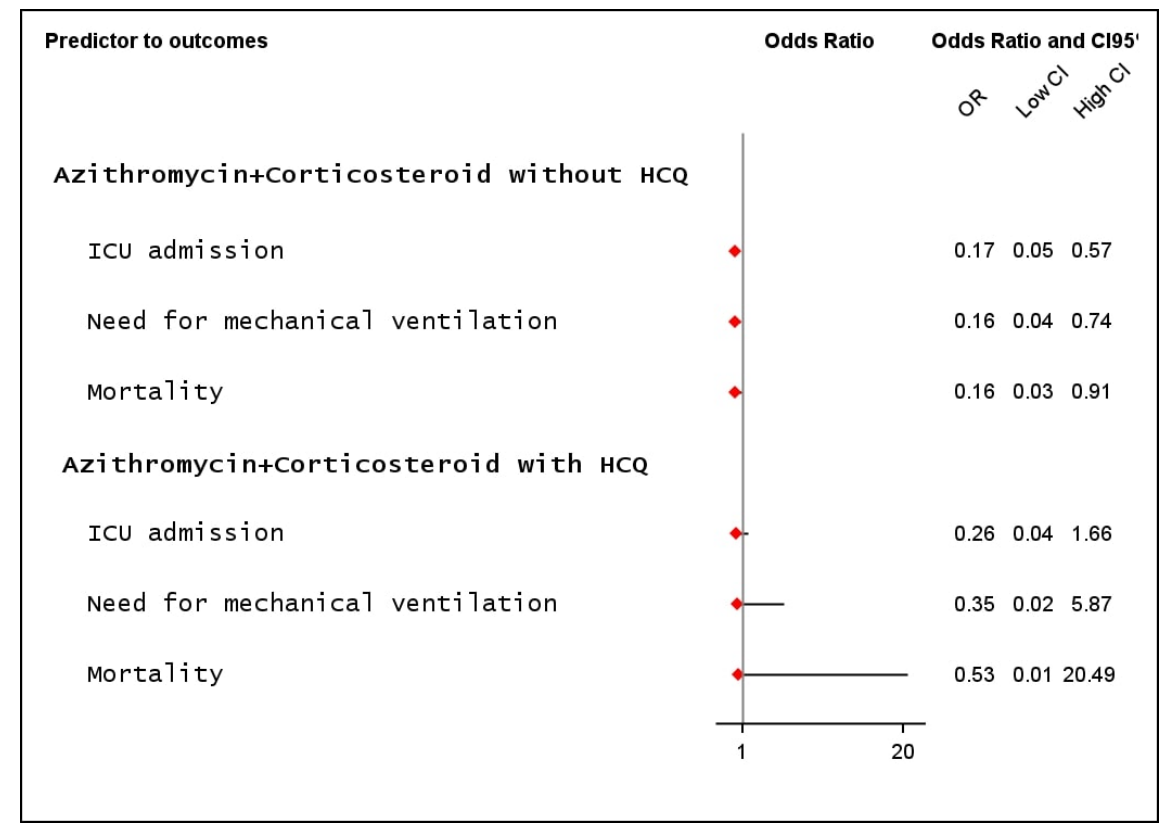




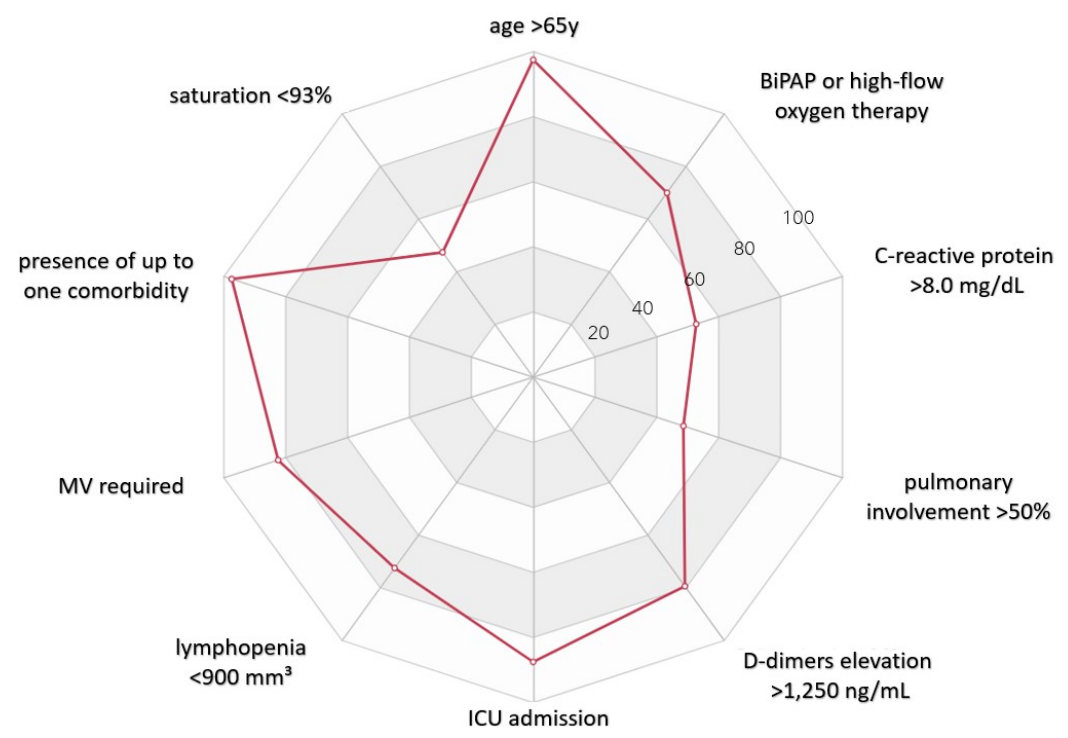

Predictor to length of stay

Beta Beta and $\mathrm{Cl} 195 \%$

Male sex

More than 65 years

obesity

At least one comorbidity

chest computed Tomography $>50 x$

C-reactive protein $>8$

D-Dimers > 1250

Nasal oxygen catheter

Hudson mask

Azithromycin+Corticosteroid

Hydroxych loroquine

Azithromycin+Corticosteroid ${ }^{*}$ Hydroxych loroquine
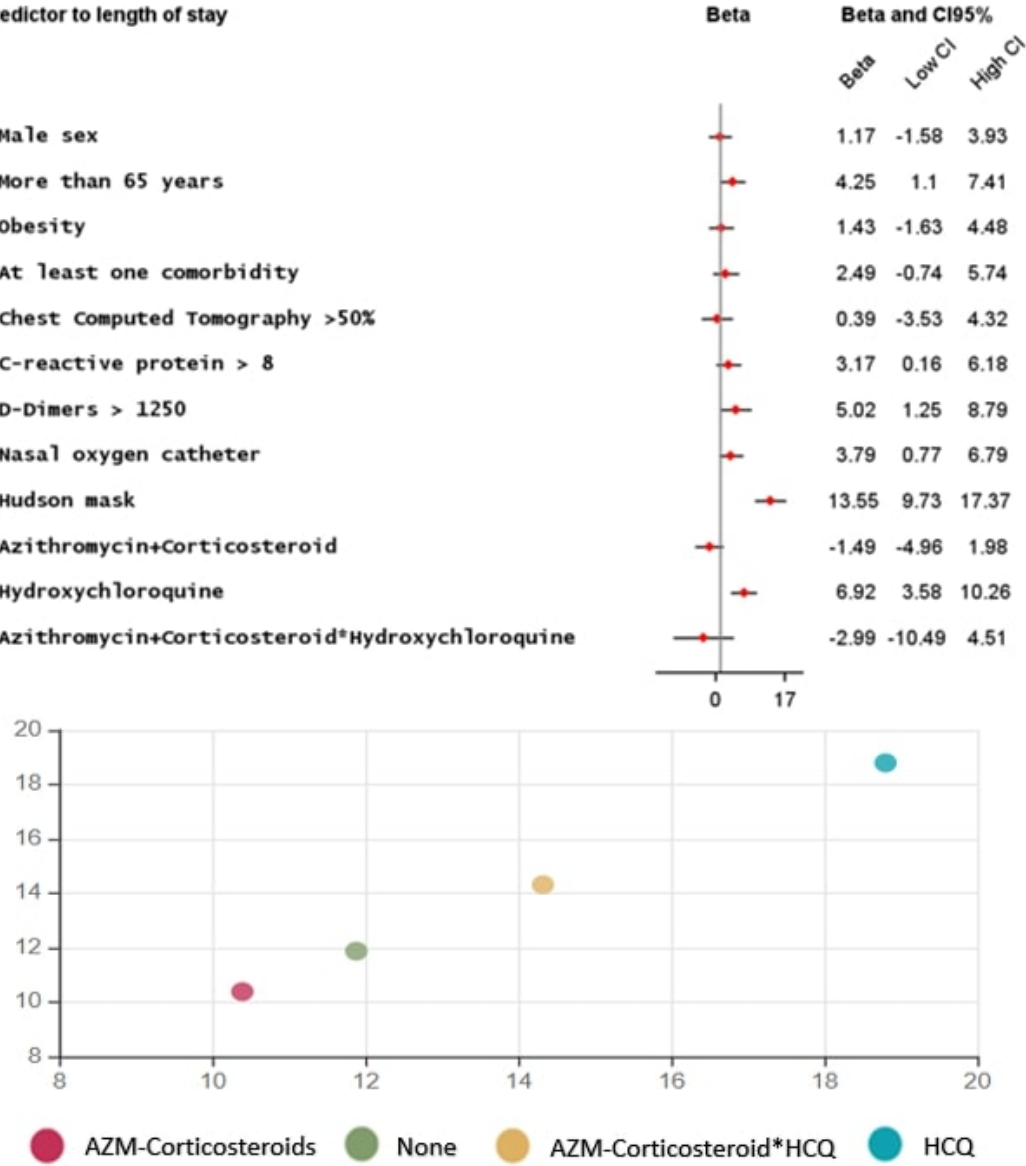
Predictor to length of stay in ICU

Beta Beta and $\mathrm{C} 195 \%$

Male sex

More than 65 years

obesity

At least one comorbidity

Chest computed Tomography $>50 \%$

C-reactive protein $>8$

D-Dimers > 1250

Nasal oxygen catheter

Hudson mask

Azithromycin+Corticosteroid+Anticoagulant

Hydroxych loroquine

Medication interaction
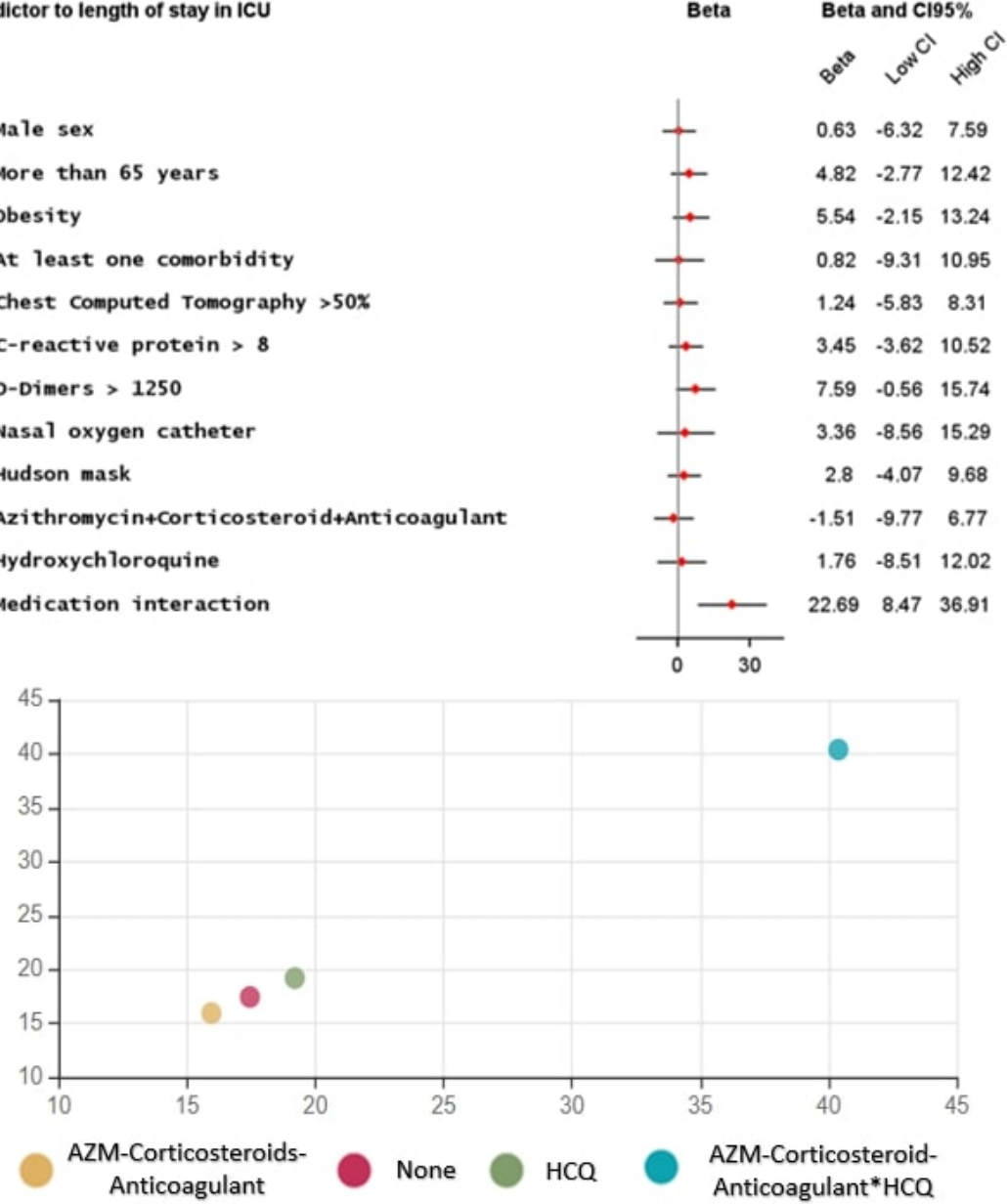
Predictor to length of stay in MV

Male sex

More than 65 years

obesity

At least one comorbidity

Chest Computed Tomography $>50 \%$

C-reactive protein $>8$

D-Dimers > 1250

Nasal oxygen catheter

Hudson mask

Azithromycin+Corticosteroid+Anticoagulant

Hydroxych loroquine

Medication interaction
Beta

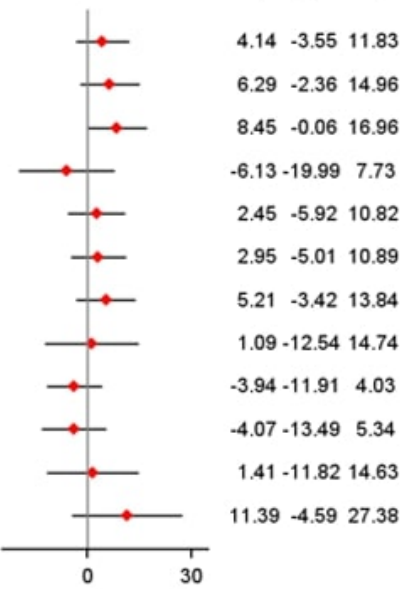

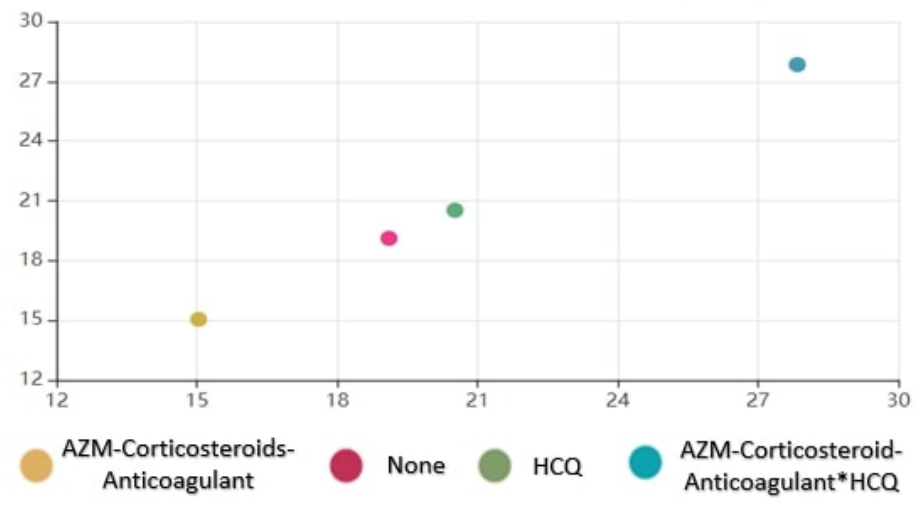

